\title{
El igualitarismo radical de John Rawls
}

\author{
JESÚS RODRÍGUEZ ZEPEDA ${ }^{1}$ \\ Universidad Autónoma Metropolitana, México
}

RESUMEN. El objetivo central de este texto es interpretar la enunciación canónica de la justicia como imparcialidad (la de Una Teoría de la Justicia) como un argumento profundamente igualitarista, incluso radical. Para ello concede especial atención a las categorías de la justicia como imparcialidad que proporcionan las señas de identidad igualitaria a su modelo liberal y, en particular, a aquellas que pretenden ofrecer una solución a los problemas de la llamada justicia social como la justa igualdad de oportunidades y el principio de diferencia. El artículo evalúa el peso relativo de cada una de estas categorías en la construcción del argumento rawlsiano a favor de la igualdad social y económica.
ABSTRACT. The main purpose of this paper is to offer an interpretation of the canonical statement of justice as fairness (A Theory of Justice) as a deeply egalitarian, even radical, argument. In order to do so, the categories of justice as fairness strongly related to the egalitarian and distributive meaning of the rawlsian liberal model and, specifically, those aimed to solve the problem of social justice, are accurately revised, namely, the fair equality of opportunity and the difference principle. The paper evaluates the relative weight of each of these categories in the rawlsian argument for social and economic equality.

El debate normativo de la igualdad es otro a partir de Rawls. Aunque durante un tiempo fueron simultáneas su propuesta de un liberalismo distributivo con las exigencias de igualdad derivadas del paradigma del socialismo revolucionario, la escasa pero intensa historia transcurrida desde 1971 (fecha de publicación de $A$ Theory of Justice) hasta nuestros días muestra que la poderosa intuición moral rawlsiana acerca de que los mecanismos de distribución económica no se pueden legitimar al margen o en contra de las libertades y derechos fundamentales de la ciudadanía democrática estaba llamada a una larga existencia, mientras que la ruta revolucionaria a la igual-

\footnotetext{
1 Profesor Investigador del Departamento de Filosofía de la Universidad Autónoma Metropolitana, campus Iztapalapa (México). Coordina el Posgrado de Filosofía Política de esa Universidad y es Investigador Nacional, nivel II, en el Sistema Nacional de Investigadores. Su últimos libros son La política del consenso: una lectura crítica de El liberalismo político de John Rawls (Barcelona, Anthropos, 2003) y Estado, publicidad y transparencia: un paseo por la filosofía política (México, IFAI, 2004). Correo electrónico: rozj@xanum.uam:mx
} 
dad estaba condenada al fracaso normativo y político. Incluso ahora, muerto Rawls, cerrada su obra y desafiada la filosofía política académica por procesos políticos de creciente complejidad, su teoría sigue siendo una piedra de toque para la discusión de la justicia, la libertad y la igualdad.

La obra de John Rawls, en este sentido, ha sido ampliamente reconocida como un punto de inflexión del pensamiento liberal, pero también en el democrático. No se trata sólo de que el renacimiento del interés por la filosofía política en las dos últimas décadas del siglo pasado haya estado invariablemente unido a su Teoría de la Justicia ${ }^{2}$, sino de que además buena parte de los conceptos y discusiones en liza en la filosofía moral y política o sigue siendo rawlsiana o está siendo versus Rawls. Los debates actuales del multiculturalismo, la política de la identidad, la política de la diferencia, las renacientes teorías de la discriminación o las nuevas teorías de la ciudadanía se construyen con el liberalismo rawlsiano como telón de fondo ${ }^{3}$.

Su reivindicación de la justicia como el tema central del pensamiento moral y político, su reformulación de la doctrina contractualista, su crítica al utilitarismo y al intuicionismo moral, su formulación - ahora canónica- de los principios de justicia característicos de una sociedad bien ordenada, su giro político en la formulación de la teoría del liberalismo político, su deriva hacia el constitucionalismo filosófico, su apuesta por el consenso como vía para la razón política son, entre otras ideas, puntos de referencia del debate moral y político de las dos últimas décadas ${ }^{4}$.

La relevancia todavía vigente de la obra de Rawls no parece estar determinada solamente por sus cualidades teóricas y su potencial explicativo;

${ }^{2}$ John Rawls, A Theory of Justice, Cambridge, Mass., publicado por President and Fellows of Harvard College, 1971. Para este trabajo he utilizado la edición paperback de Oxford University Press, 1973.

${ }^{3}$ Will Kymlicka señalaba en el ya lejano 1989 que el liberalismo de referencia para la discusión de la filosofía política sólo podía ser el liberalismo normativo de corte rawlsiano, diferente del liberalismo del siglo XIX, y que para algunos propósitos podría ser denominado, como sugiere G. A. Cohen, nuevo liberalismo o liberalismo socialdemócrata. Véase W. Kymlicka, Liberalism, Community and Culture, Oxford, Clarendon Press, 1989, pp. 9-10.

${ }^{4}$ Para tener una idea más precisa de esta influencia de Rawls en las filosofías moral y política, véase Samuel Friedman (ed.), The Cambridge Companion to Rawls, Cambridge, UK, Cambridge University Press, 2003; también Pablo de Silveira y Wayne Norman, «Rawlsianismo metodológico: una introducción a la metodología dominante en la filosofía política anglosajona contemporánea», Revista Internacional de Filosofía Política, núm. 5, UNED-UAM, junio de 1995. Para documentar el papel de Rawls como «resucitador» de la filosofía política, véanse como muestra Peter Laslett y James Fishkin (comps.), Philosophy, Politics and Society, Fifth Series, Oxford, Basil Blackwell, 1979; Brian Barry, Theories of Justice, vol. 1, Londres, Harvester-Wheatsheaf, 1989; Kukathas y Pettit, Rawls: «A Theory of Justice» and its Critics, Cambridge, Polity Press, 1990, y David Boucher y Paul Kelly (comps.), The Social Contract from Hobbes to Rawls, Londres, Routledge, 1994. Pero acaso el mayor logro de Rawls haya sido el de contribuir a cerrar la brecha entre la filosofía política continental, magníficamente representada en la obra de Jürgen Habermas, y la anglosajona, que él mismo coronó con solvencia. Véanse J. Habermas y J. Rawls, Debate sobre el liberalismo político (ed. de Fernando Vallespín), Barcelona, Paidós-ICE de la Universidad Autónoma de Barcelona, 1998. 
está informada también por el perfil político que sus principales conceptos poseen en relación con un nuevo intento de justificación del Estado de bienestar y de los sistemas democráticos en general. En efecto, aunque la Teoría de la Justicia fue publicada en una época en la que, para muchos, aún prevalecían las esperanzas de fundamentar los proyectos de cambio social en alguna de las versiones de la teoría marxista, lo cierto es que el paulatino deterioro de los ensayos de socialismo real, cuya brusca culminación a finales de los ochenta fue no obstante sorpresiva, y el fracaso político rotundo de los celebrados en su momento Movimientos de Liberación Nacional, entre otros procesos similares, empezaron a dejar sin oponentes reales a la entonces denostada democracia liberal. En este sentido, la teoría rawlsiana de la justicia ha venido a representar, al menos para muchos circuitos académicos, tanto una reivindicación de los valores liberal-democráticos, tantas veces malentendidos o de plano desconocidos, como un compromiso con la justicia distributiva que las visiones liberales al uso habían postergado con mucha frecuencia. Por ello, la llamada justicia como imparcialidad (justice as fairness) ${ }^{5}$ puede presentarse legítimamente como el intento más sistemático de los últimos tiempos de alcanzar el ideal de equilibrio entre las llamadas libertad negativa y positiva o, dicho en otros términos, entre las garantías de los ciudadanos de no interferencia estatal respecto de su vida y proyectos y el compromiso moral y político con la participación política, la nivelación social y la reducción de las desigualdades económicas. En consecuencia, la justicia como imparcialidad debe ser vista no sólo como un modelo de coherencia entre las libertades positiva y negativa, sino también como un intento de equilibrar los siempre conflictivos principios de libertad individual e igualdad material.

Las interpretaciones más frecuentes del proyecto rawlsiano lo identifican con un modelo de sociedad plural-democrática, capitalista y altamente distributiva en el que es posible la vigencia de los derechos y libertades ciudadanos fundamentales bajo la égida de los principios de libertad e igualdad. No obstante, existen otros puntos de vista que sostienen que las condiciones distributivas establecidas por una sociedad bien ordenada guiada por los principios de la justicia difícilmente podrían ajustarse al marco de institucio-

\footnotetext{
5 He optado por mantener la traducción de fairness por «imparcialidad» según se presentó en la edición del Fondo de Cultura Económica. Otra traducción posible de fairness, la de «equidad», me parece más adecuada (cf. la selección y traducción de Miguel Ángel Rodilla de una serie de materiales previos y posteriores a la Teoría de la Justicia bajo el título de, precisamente, Justicia como equidad, Madrid, Tecnos, 1986). Sin embargo, aunque discutible, la primera acepción se ha hecho canónica, lo que no obsta para que siga dando lugar a numerosos problemas para el lector en español. Por ejemplo, en el libro de Brian Barry, Justice as Imparciality, Oxford, Oxford University Press, 1995 (traducido, naturalmente, como La justicia como imparcialidad, Paidós, Barcelona, 1997), se presenta al modelo rawlsiano de justicia (justice as fairness) como uno más de los casos de la justicia como imparcialidad -en contraste con lo que el propio Barry llama la concepción de la justicia como provecho mutuo-, por lo que un lector desprevenido de las versiones en español de los libros de Rawls y Barry se empantanaría en una gratuita confusión terminologica.
} 
nes de una sociedad propiamente capitalista y tendrían, por ende, que conducir a algún modelo de socialismo democrático ${ }^{6}$. Incluso, de manera sorprendente, existen interpretaciones que pretenden establecer una continuidad entre la justicia como imparcialidad y los puntos de vista filomercantiles del neoliberalismo en sus versiones más extremas ${ }^{7}$. Aunque esta problemática invoca algunas cuestiones adicionales, como la definición misma de lo que es una sociedad de mercado, lo cierto es que las interpretaciones más consistentes de la obra de Rawls, así como la propia autodefinición de este autor, sitúan su proyecto en el terreno de las argumentaciones en favor de la reforma del Estado de bienestar y en modo alguno en su supresión o superación definitiva.

El objetivo central de este texto es defender el carácter radical del igualitarismo rawlsiano de la primera época, el de la enunciación canónica de la justicia como imparcialidad (aunque luego hayamos sabido por el propio Rawls que se trataba de una formulación comprehensiva que merecía una revisión política). Por ello, es necesario conceder especial atención a aquellas categorías de su justicia como imparcialidad que proporcionan las señas de identidad igualitaria a su modelo liberal y, en particular, a aquellas que pretenden ofrecer una solución a los problemas de la llamada justicia social como la justa igualdad de oportunidades y el principio de diferencia.

Rawls concede los beneficios de una distribución absolutamente equitativa sólo al conjunto de libertades y derechos que constituyen la ciudadanía

${ }^{6}$ Las interpretaciones que ven en Rawls un teórico del Estado capitalista de bienestar cuentan entre sus filas a los siguientes autores: Robert Paul Wolff, Understanding Rawls: A Reconstruction and Critique of «A Theory of Justice», Princeton, NY, Princeton University Press, 1977; Brian Barry, The Liberal Theory of Justice, Nueva York, Oxford University Press, 1973; Norman Daniels (ed.), Reading Rawls, Nueva York, Basic Books, 1975; Amy Gutmann, Liberal Equality, Nueva York, Cambridge University Press, 1980, y «Rawls on the Relationship between Liberalism and Democracy», en Sanuel Freeman (ed.), The Cambridge Companion to Rawls, ed. cit., pp. 168199, y Alan Ryan, Property in Political Theory, Nueva York, Basic Blackwell, 1984. Para acercarse a una interpretación que hace del modelo social de Rawls un proyecto socialista democrático, véanse Arthur di Quattro, «Rawls and Left Criticism», Political Theory, núm. 2, febrero de 1983, pp. 54-78, y Richard Krouse y Michael McPherson, «Capitalism, "Property-Owning Democracy", and the Welfare State», en Amy Gutmann (ed.), Democracy and the Welfare State, Princeton, Nueva Jersey, Princeton University Press, 1988.

7 No es desconocida la interpretación de Nozick en su Anarquía, Estado y utopía (México, FCE, 1988), según la cual la obra de Rawls habría instalado el campo de discusión de la filosofía política contemporánea y, más particularmente, que la propia teoría de un Estado mínimo no sería incompatible con el neocontractualismo rawlsiano (cf. pp. 40 ss., 45 y ss., y 183 ss.). Realmente sorprendente es la interpretación de Hayek, quien dice discrepar de Rawls «sólo» en el uso del término justicia social, y coincidir con él en que «"los principios relativos a la justicia definen más bien ciertas condiciones que es preciso imponer a las instituciones y al comportamiento colectivo al objeto de que éstas puedan ser por las gentes respaldadas. Si tales condiciones quedan satisfechas, la distribución resultante, fuere la que fuere, deberá ser considerada justa..." Y - agrega Hayek- en términos generales, ésta es la conclusión hacia la que [...] he intentado llevar el ánimo del lector». Friedrich A. Hayek, Derecho, legislación y libertad, vol. II, Madrid, Unión Editorial, 1988, p. 182. La cita de Rawls proviene de John Rawls, «Constitucional Liberty and the Concept of Justice», en Nomos, IV, Justice, Nueva York, 1963, p. 102. 
democrática, mientras propone un modelo de desigualdad «regulada», «controlada» o «razonable» para la distribución de la riqueza, el ingreso y las posiciones sociales relacionados con éstos. Ello es el resultado de la consideración de que determinadas diferencias en la repartición del ingreso, la riqueza y las posiciones con ellos relacionados pueden ser justificadas si, tomando como punto de comparación la posición menos aventajada, trabajan en favor de las posiciones peor situadas.

En todo caso, el valor político de la igualdad recibe de Rawls un tratamiento inusual dentro de la tradición discursiva liberal, pues no sólo se admite, contra la disyuntiva obligada entre libertad e igualdad que había hecho canónica Isaiah Berlin desde los años veinte del siglo pasado, que es posible una sociedad liberal puesta, por vía «reformista», en camino de la igualdad económica, sino que se postula la necesidad de justificar formas determinadas de desigualdad en la distribución material cuando sirven al propósito de favorecer las posiciones menos aventajadas.

Esta pretensión de dar cobertura moral a determinadas desigualdades abrió un flanco de crítica a Rawls por caer supuestamente en la defensa de un falso igualitarismo en el terreno económico y, en consecuencia, por una posible recaída en la vieja unilateralidad liberal de garantizar sólo una igualdad formal de derechos que estaría escondiendo, y en ese sentido justificando, una desigualdad material a la que no se atreve a condenar, pero que mina las posibilidades de realización de los propios derechos y libertades de la ciudadanía ${ }^{8}$. Un apresurado dictamen sobre la cuestión igualitaria en Rawls llevaría a decir que, puesto que no se puede garantizar una igualdad absoluta en el terreno de las instituciones y relaciones económicas y no se propone la sociedad bien ordenada como negación estructural del capitalismo, la igualdad liberal estaría fallando al enfrentar las injusticias generadas por una sociedad capitalista de mercado.

Es cierto que el propio Rawls se negó a sostener tanto un igualitarismo total en el campo del ingreso y la riqueza como la deseabilidad de la desaparición del capitalismo; pero esto no implica que se haya quedado corto respecto de las posibilidades distributivas de su modelo de justicia social. Aun más, creo que puede afirmarse que la de Rawls es una de las versiones distribucionistas más radicales que pueden formularse desde el requisito de la inviolabilidad de los derechos y libertades ciudadanas. El mérito de esta for-

\footnotetext{
${ }^{8}$ Ésa es la postura de C. B. Macpherson, quien al argumentar la diferencia entre su posición y la de Rawls señala lo siguiente: «Por supuesto, son posibles modelos no capitalistas que utilicen 0 permitan diferencias de ingreso o riqueza, y éstos pueden redundar en [la existencia de] clases según el ingreso y la riqueza. Pero estas últimas no necesitan crear derechos y libertades desiguales dado que no son el resultado o los medios de la dominación ni obtienen ganancias a expensas de las demás. Los principios de la justicia de Rawls, aplicados a ese tipo de sociedad, probablemente tendrían mucho sentido. Sin embargo, él los aplica a una sociedad capitalista, donde se convierten en una carta de autorización para el liberalismo revisionista», Democratic Theory, Nueva York, Oxford University Press, p. 90.
} 
mulación puede hacerse más notorio si se ponen en cuestión las posibilidades efectivas y, más importante todavía, la deseabilidad de un igualitarismo completo en el terreno económico en el marco de una sociedad pluralista. Significativamente, incluso las más radicales entre las demandas de nivelación económica que podrían ser democráticamente justificadas en nuestra época tienen que estar emparentadas en primer grado con los principios rawlsianos de diferencia y de igualdad justa de oportunidades 9 .

En este contexto, no veo razones para no conceder a la teoría rawlsiana el calificativo de igualitaria en el terreno económico y, sobre todo, de radicalmente igualitaria, toda vez que ofrece un modelo distributivo guiado normativamente por el supuesto de la igualdad de las personas. Creo que esto explica el que Rawls suponga, en algunos casos, el concepto de igualdad ( $\mathrm{y}$ no el de desigualdad controlada o limitada) como punto de referencia para medir las diferencias legítimas en la distribución del ingreso y la riqueza ${ }^{10}$.

En este sentido, si puede uno hacerse cargo de la idea de que es posible la justificación de determinadas desigualdades económicas en razón del bienestar de todas las partes y, particularmente, de las peor situadas, tendríamos un criterio para rechazar las desigualdades económicas arbitrarias (que sólo benefician a algunos) y la posibilidad de diseñar políticas públicas sensatas (pero radicales) para situar a la sociedad en la «tendencia a la igualdad». En efecto, las medidas distributivas determinadas por el principio de diferencia y por la igualdad de oportunidades, en cuanto permiten resarcir a las posiciones peor situadas por las desventajas inmerecidas, establecen una tendencia a la convergencia de las posiciones sociales $y$, en este sentido, a la de una igualdad relativa que previene la inestabilidad y fragmentación sociales.

La noción de igualdad posee, en este sentido, una multidimensionalidad que la haría irreductible a sólo alguno de los niveles de análisis en los que se desarrolla la justicia como imparcialidad. Por ello, para Rawls, la igualdad presenta al menos tres niveles de aplicación:

El primero es el de la administración de las instituciones en tanto que un sistema público de leyes. En este caso, igualdad es esencialmente justicia como regularidad [...]. La igualdad a este nivel es el elemento menos controvertido para una noción de sentido común de la justicia. La segunda y mucho más ardua aplicación de la igualdad se da para el caso de la estructura sustantiva de las instituciones. Aquí, el significado de la igualdad es especificado por los principios de la justicia que requieren

\footnotetext{
9 Resulta interesante, por ejemplo, la relación que establece Philippe Van Parijs entre su idea de la renta universal básica y una interpretación del principio rawlsiano de diferencia. Cf. P. van Parijs, «Difference Principles», en S. Freeman, The Cambridge Companion to Rawls, ed. cit., pp. 200-240.

10 Por ejemplo, en una descripción de la posición originaria, dice Rawls: «... las partes inician con un principio que establece una libertad equitativa para todos, lo que incluye la igualdad de oportunidades, y una distribución equitativa del ingreso y la riqueza», $T J$, p. 151 . Pero en seguida ofrece las razones para abandonar la igualdad en el último terreno si puede establecerse un principio que proporcione, regulando esos desniveles, beneficios a todas las partes. Cf. ibid.
} 
la asignación de derechos básicos equitativos a todas las personas [...] Esto nos lleva al tercer nivel, en que aparece el tema de la igualdad [...] son precisamente las personas morales las que poseen el derecho a una justicia equitativa... La justicia equitativa es debida a aquellos que tienen la capacidad de tomar parte en, y actuar de acuerdo con, el entendimiento público de la situación inicial ${ }^{11}$.

Tomaré los dos primeros niveles de esta clasificación para abundar en los principios de igualdad de oportunidades y de diferencia, en el entendido de que el tercer nivel, el de la igualdad como postulado moral, nos lleva bastante lejos del tema de la distribución económica.

La primera de estas nociones, como señala el propio Rawls, es, en efecto, la menos problemática de las tres en cuanto a su concepción y aplicación. Su definición puede ofrecerse bajo el marco de la fórmula de la «justicia puramente procedimental» (pure procedural justice). Este tipo de justicia, a diferencia de la justicia procedimental perfecta (que supone la apelación a un principio previo y externo al procedimiento que habrá de seguirse) o de la justicia procedimental imperfecta (que supone un criterio independiente para la definición del resultado correcto pero no puede garantizar un procedimiento correcto que permita llegar a él), se caracteriza porque el criterio para la definición del resultado correcto no es independiente del procedimiento para llegar a él ${ }^{12}$. Se trata, entonces, de un modelo de justicia en el que la validez de los resultados del procedimiento (en el caso de Rawls, la validez de los principios de la justicia y de las instituciones y normas fundadas en ellos) depende de un principio rector que se formula sólo a través de, y a la par que, el procedimiento (en este caso, la concurrencia contractual de personas libres e iguales). Rawls pretende que la justicia como imparcialidad debe funcionar, precisamente, como un modelo de justicia puramente procedimental. No obstante, no mantiene que el procedimiento por sí mismo pudiera dar lugar a la imparcialidad, sino que este modelo de igualdad formal sólo tiene sentido si está soportado y actualizado por un conjunto de instituciones y un sistema constitucional realmente equitativos. En este sentido, por ejemplo, no basta, para alcanzar la vigencia de la justicia en una sociedad, con que los preceptos legales sean aplicados bajo modalidades sujetas a tiempos y formas determinados y que estén encuadrados en un sistema formal coherente; hace falta que estos procedimientos legales actualicen un modelo legal democráticamente diseñado (es decir, dialógica y racionalmente alcanzado). De este modo, se requiere que la justicia como imparcialidad sea procedimentalmente pura, pero, institucional y políticamente, libre y distributiva.

\footnotetext{
11 TJ, pp. 504-505.

12 Dice Rawls: «Un rasgo característico de la justicia puramente procedimental consiste en que el procedimiento que determina el resultado justo debe ser realmente llevado a cabo; así que en estos casos no existe un criterio independiente por referencia al cual se pueda saber que un resultado particular es justo», $T J$, p. 86 .
} 
Decir que una sociedad es justa es decir que funciona en ambos niveles. El modelo rawlsiano de justicia no podría ser perfectamente procedimental porque con ello escamotearía a las personas su derecho a definir las reglas de justicia que han de determinar las distribuciones que les atañen. Tampoco debe ser un modelo procedimental imperfecto, pues, de serlo, no podría entonces garantizar que su método de distribución arroje siempre el mismo tipo de resultados; sobre todo porque la distribución no puede ser, respecto de los mismos sujetos y las mismas situaciones, justa e injusta de manera sucesiva.

En este sentido, la justicia puramente procedimental tendría la ventaja de garantizar, siempre que como método se aplique con regularidad y sin excepciones significativas, el mismo tipo de consecuencias distributivas. Si a ello se suma que este modelo de justicia actualiza los principios constitucionales y normativas institucionales de una sociedad bien ordenada, tenemos ya un modelo de distribución que conjunta igualdad democrática (tanto en su confección como en su aplicación) y reducción de la incertidumbre en el terreno distributivo.

Esta distinción entre distintas fórmulas de justicia y la elección de la justicia puramente procedimental como la que satisface las expectativas de la justicia como imparcialidad constituyen una perspectiva coherente para situar el segundo nivel de aplicación de la noción de igualdad, a saber, el de la especificación de la naturaleza y alcance de los principios de la justicia en la estructura básica de la sociedad. En este terreno podemos abordar en forma serial el desenvolvimiento del requisito de igualdad según se va aplicando a cada principio de la justicia ${ }^{13}$.

En el caso del terreno de aplicación del primer principio está supuesta una equitativa distribución de los derechos y libertades característicos de la ciudadanía democrática; por ello, lo que se presenta como problemático es, más bien, el caso del territorio sujeto a la cobertura del segundo principio. El orden serial de los principios de la justicia, es decir, la secuencia lexicográfica que exige el cumplimiento de los preceptos formulados en primera instancia para la viabilidad moral de los formulados posteriormente, establece una continuidad entre el primer principio de la justicia — referido al conjun-

${ }^{13}$ La canónica de estos principios es la de la primera parte de $A$ Theory of Justice: «Primero: cada persona ha de tener un derecho igual a la más extensa libertad básica compatible con una libertad similar para todos. Segundo: las desigualdades sociales y económicas han de ser ordenadas de tal forma que: $a$ ) se espere razonablemente sean ventajosas para todos, y $b$ ) estén vinculadas a empleos y cargos asequibles para todos» (p. 60). Otras formulaciones de los principios, con correcciones y agregados menores, pueden encontrarse, entre otros lugares, en las pp. 83, 250 y 302-303 del mismo libro. En una versión posterior, los principios se formularon así: «1. Cada persona tiene igual derecho a un esquema completamente adecuado de libertades básicas iguales, compatible con un esquema de libertades similar para todos. 2. Las desigualdades sociales y económicas han de cumplir dos condiciones. Primera, deben estar vinculadas a oficios y posiciones abiertas a todos bajo condiciones de justa igualdad de oportunidades; y segunda, deben operar para el mayor beneficio de los miembros menos aventajados de la sociedad.» John Rawls, Political Liberalism, Columbia University Press, 1993, p. 291. 
to de libertades básicas - y la primera parte del segundo principio - que, con la aplicación del criterio lexicográfico - vendría a ser la justa igualdad de oportunidades ${ }^{14}$. Esta continuidad puede notarse en la inclusión de la justa igualdad de oportunidades como uno de los bienes primarios cuyo acceso equitativo debe estar garantizado a todos los sujetos ${ }^{15}$.

En este sentido, la igualdad de oportunidades no puede sujetarse internamente al principio de diferencia pese a que, a nivel práctico, su condición equitativa ideal no pudiera satisfacerse dada la diversidad natural de los seres humanos; aunque sí podría beneficiarse de los equilibrios sistémicos introducidos por aquél. Dicho de otro modo, no es posible la aplicación del principio de igualdad de oportunidades para regular las diferencias entre las capacidades y talentos naturales que condicionan una desigual participación en las porciones de riqueza e ingreso. No obstante, su posición intermedia entre el sistema de libertades ciudadanas y el principio regulador de las diferencias económicas exige que se mantenga, simultáneamente, su aplicación universal y la necesidad de compensar sus limitaciones. Si consideramos que los desniveles resultantes de la imperfecta aplicación de una justa igualdad de oportunidades pueden ser equilibrados por la aplicación del principio de diferencia, podremos entender que esta prerrogativa social quede fuera del elenco de libertades básicas, ya que incluirla en éste habría violado el principio de prioridad e inafectabilidad de la libertad respecto de las condiciones económicas de la sociedad. Pero si se considera que la igualdad de oportunidades es un requisito para la consecución de los objetivos morales de la justicia como imparcialidad, no podemos menos que conceder que su formulación como bien de distribución homogénea es ineludible.

La estrategia discursiva de Rawls para solventar el problema de la formulación de una noción de igualdad de oportunidades congruente con los requisitos igualitarios de la justicia como imparcialidad descansa, más que cualquier otro pasaje de su Teoría de la Justicia, en consideraciones históricas y políticas. La preferencia por la noción de «igualdad democrática de oportunidades», como una conclusión necesaria tras la evaluación de nociones alternativas como las del «sistema de libertad natural» y la «interpretación liberal», muestra el interés de Rawls por escapar a las limitaciones que conlleva la enunciación formal de la igualdad de oportunidades ${ }^{16}$. Es claro que Rawls pudo haber argumentado al respecto tal como lo hizo a propósito de las liber-

14 En su formulación revisada de los principios de la justicia y de las reglas de prioridad, Rawls dice que «... la igualdad de oportunidades es prioritaria respecto del principio de diferencia», $T J$, pp. 302-303. Rawls lo esquematiza de la siguiente manera en la p. 124:

«Los dos principios de la justicia (en orden serial):

1. Los principios de la más amplia libertad equitativa.

2. a) El principio de (justa) igualdad de oportunidades;

b) el principio de diferencia.»

15 Cf. $T J$, p. 93

16 Cf. $T J$, pp. $72-75$. 
tades básicas, dando por sentada su distribución homogénea y aceptando variaciones de ella sólo en el terreno de sus valoraciones subjetivas. En este sentido, pudo haber solventado el tema con la postulación de la igualdad de oportunidades como un derecho de disfrute universal (lo que ciertamente es el argumento de casi toda la tradición liberal) cuyos desniveles provendrían de consideraciones valorativas y no de su realidad institucional. ¿Quién podría negar que en los sistemas legales, modelos institucionales y discursos políticos de los países liberales la igualdad de oportunidades es apreciada como un pilar básico de la vida colectiva? ¿Por qué habría que considerar alguna diferencia entre la igualdad de oportunidades y, digamos, la libertad de expresión o los derechos políticos? Creo que la respuesta a esta «desviación» de Rawls respecto de la ortodoxia liberal reside, precisamente, en su negativa a respaldar el formalismo de las defensas al uso de la igualdad de oportunidades. Incluso creo que el que el imaginario colectivo norteamericano haya hecho de la idea de igualdad de oportunidades uno de sus valores ideológicos centrales previene a Rawls de un tratamiento superficial de esta cuestión ${ }^{17}$. En este sentido, la propuesta de la igualdad justa de oportunidades muestra la preocupación de Rawls por mantener el delicado equilibrio entre nivelación económica y derechos de ciudadanía, característico de su teoría de la justicia, sin ceder a la tentación del formalismo liberal.

Con el rechazo a la igualdad de oportunidades concebida sólo como libre acceso formal a las posiciones sociales privilegiadas, Rawls se desmarca con claridad del enunciado moral básico del neoliberalismo, a saber, la postulación de un sistema de libertad natural, actualizado en las relaciones de mercado, que entiende la justicia como el respeto a la colocación de los recursos y las posiciones sociales que son el resultado de un libre intercambio generalizado, por lo que cualquier distribución gubernamental o estatalmente inducida vendría a ser injusta ${ }^{18}$. La brillante sugerencia de Rawls, implícita

17 William Galston argumenta que los dos pilares de una sociedad liberal moderna son la distribución de los bienes sobre la base de las necesidades equitativamente consideradas y la adjudicación de oportunidades sociales según un criterio de derecho equitativo de participación. Así, «el último principio (derecho equitativo de participación) ingresó en el pensamiento político norteamericano bajo la rúbrica de "igualdad de oportunidades". Gran parte de la historia social norteamericana puede ser interpretada como una lucha entre los que deseaban ampliar el alcance de su aplicación y los que buscaban restringirlo». William Galston, «Equality of Opportunity and Liberal Theory», en Frank S. Lucash (comp.), Justice and Equality: Here and Now, Ithaca y Londres, Cornell University Press, 1986, p. 89. Por supuesto, Rawls estaría situado entre los miembros del primer grupo.

18 Una clara defensa de esta posición está en F. A. Hayeck. Según él, «la justicia, en efecto, requiere que aquellas condiciones de la vida de las personas que son determinadas por el gobierno sean provistas por igual a todos. Pero la igualdad de esas condiciones debe conducir a la desigualdad de resultados [...]. Sin embargo, es una cuestión del todo diferente sugerir que aquellos que son pobres, meramente en el sentido de que hay otros en la misma comunidad que son ricos, tienen derecho a una porción de la riqueza de estos últimos, o que el haber nacido en un grupo que ha alcanzado un nivel particular de civilización y comodidad les confiera el derecho a una parte de todos sus beneficios». F. A. Hayek, The Constitution of Liberty, Londres, Routledge and Kegan Paul, 1963 , pp. 99 y 101 . 
en este argumento, acerca de que la justicia no puede ser el resultado de un proceso natural, es decir, de una serie de hechos dados arbitrariamente, descarta claramente la idea de que un sistema de justicia moralmente justificable pudiera ser el resultado espontáneo de una estructura mercantil ${ }^{19}$. Esta negación es, precisamente, la base para justificar los procesos de nivelación que garanticen una efectiva igualdad de oportunidades. La innovación de esta posición respecto, por ejemplo, de la crítica marxista consiste en que las diferencias económicas de poder y rango pueden ser tachadas de injustas incluso bajo el supuesto de que fueran el resultado del ejercicio de los talentos y capacidades naturales de las partes y sin que hubiera mediado violencia alguna en su constitución histórica. En este sentido, la crítica rawlsiana al supuesto neoliberal de la igualdad formal de oportunidades es más coherente con la historia reciente de los países capitalistas, donde la regularidad del proceso económico y del Estado de Derecho en las últimas décadas parece conceder carácter contractual (por medio de una aceptación tácita) a las graves diferencias sociales vigentes.

La concepción alternativa al llamado sistema de libertad natural es la que Rawls denomina «igualdad liberal». La igualdad liberal de oportunidades trata de compensar todas las desigualdades que dan lugar a que prevalezcan las ventajas de unas posiciones sociales sobre otras, es decir, añade el principio de justa igualdad de oportunidades (fair equality of opportunity) al principio de trayectorias profesionales abiertas del sistema de libertad natural. Según este nuevo principio, un sistema de justicia debe propiciar que la igualdad formal de acceso a las posiciones privilegiadas pueda ser aprovechada por todas las posiciones. Esto implica que las expectativas de quienes poseen similares capacidades y talentos y tienen, además, similar disposición para ejercitarlos deben tener las mismas oportunidades independientemente de su clase social. Esta nivelación en las oportunidades exige, en la perspectiva de Rawls, la promoción de las posiciones socialmente peor situadas. Según Rawls, esta interpretación pretende

... mitigar la influencia de las contingencias sociales y de la fortuna en las porciones distributivas. Para alcanzar este objetivo es necesario añadir condiciones estructurales básicas al sistema social. Los circuitos del mercado libre deben ser enmarcados por un esquema de instituciones políticas y legales que regulen las tendencias generales de los hechos económicos y preserven las condiciones sociales necesarias para la justa igualdad de oportunidades. Los elementos de este esquema son suficientemente conocidos, pero vale la pena recordar la importancia de la prevención de acumulaciones excesivas de propiedad y riqueza y del mantenimiento de oportunidades educativas iguales para todos ${ }^{20}$.

19 Esto es muy claro en la siguiente opinión de Rawls: «La distribución natural no es justa ni injusta; tampoco es injusto haber nacido en alguna posición particular: éstos son, simplemente, hechos naturales. Lo que es justo o injusto es el modo en que las instituciones enfrentan estos hechos», A Theory of Justice, p. 102.

${ }^{20} \mathrm{TJ}, \mathrm{p} .73$ 
La propuesta liberal de una justa igualdad de oportunidades introduce la exigencia de compensar la desigualdad proveniente de las contingencias sociales por medio de políticas de promoción y afirmación de las posiciones sociales menos favorecidas.

No es difícil ver en este modelo de igualdad los principios que guían, en general, la filosofía del Estado de bienestar y, en particular, las instituciones y políticas del llamado Welfare norteamericano. Las acciones públicas orientadas hacia el equilibrio en la distribución de la riqueza y el ingreso públicos a través de una política fiscal muy exigente, los programas gratuitos de salud pública, la ampliación de las expectativas profesionales y vitales derivados de un sistema educativo incluyente y gratuito, el apoyo a los grupos marginados y comunidades étnicas secularmente discriminados a través de la llamada acción afirmativa (affirmative action), la protección económica frente a las adversidades laborales como los sistemas de seguro de desempleo y pensiones son, en este contexto, los elementos característicos de este modelo de igualdad de oportunidades ${ }^{21}$. No obstante sus ventajas evidentes respecto del primer modelo de igualdad —el del sistema de libertad natural - en cuanto a la nivelación de las posiciones de partida para la competencia social y no sólo en los puntos de llegada de las trayectorias laborales, Rawls argumenta que la igualdad liberal deja sin resolver el problema de la desigualdad en la distribución de los haberes naturales (natural assets) que, situada en un proceso continuo de funcionamiento económico, sigue determinando notables disparidades en la riqueza y el ingreso.

En este sentido, el calificativo de «moralmente arbitrarias» usado para caracterizar a las desigualdades provenientes de la sociedad o la dotación natural tiene, fundamentalmente, un propósito distributivo. Éste no consiste sólo en defender la idea de que la oferta de justa igualdad de oportunidades debería ser un principio elegido por las partes de un contrato originario en persecución de sus propios intereses (lo cual es acaso la parte menos relevante del argumento), sino además en mantener que toda persona tiene derecho a una justa igualdad de oportunidades y, cuando ésta no elimina las diferencias socialmente significativas, a la compensación generada por el principio de diferencia, en razón de su naturaleza moral como persona libre e igual. En este sentido, las diferencias sociales serán arbitrarias siempre que su existencia no esté justificada por el respeto y promoción de la libertad e igualdad humanas. Creo que esto constituye el núcleo moral de la propuesta rawlsiana de igualdad democrática:

21 Cf. Amy Gutmann, «Introduction», en Amy Gutmann (comp.), Democracy and the Welfare State, ed. cit., pp. 3-12, y Robert E. Goodin, «Reasons for Welfare. Economic, Sociological, and Political - but Ultimately Moral», en Donald Moon (comp.), Responsability, Rights and Welfare. The Theory of Welfare State, Boulder y Londres, Westview Press, 1988, pp. 21-42. Véase también John E. Roemer, Equality of Opportunity, Cambridge, Mass., y Londres, Harvard University Press, 1998. 
La interpretación democrática es alcanzada cuando se combina la justa igualdad de oportunidades con el principio de diferencia [...]. Aceptando el esquema de instituciones requerido por una libertad igual y una justa igualdad de oportunidades, las más altas expectativas de los mejor situados serán justas si y sólo si funcionan como parte de un esquema que mejora las expectativas de los miembros de la sociedad menos aventajados. La idea intuitiva consiste en que el orden social no ha de establecer y asegurar las posibilidades más atractivas de los más acomodados a menos que el hacerlo redunde en la ventaja de los menos afortunados ${ }^{22}$.

La igualdad democrática defendida por Rawls, que es, como se ha visto, bastante más que la mera distribución homogénea de las libertades y derechos de la ciudadanía democrática, plantea una exigencia distributiva muy alta. La defensa de la legitimidad de ciertas diferencias económicas bajo el requisito de que contribuyan al bienestar colectivo muestra la originalidad de la posición rawlsiana respecto de las defensas tradicionales del Estado de bienestar. En su perspectiva, la justa igualdad de oportunidades, la llamada igualdad liberal, es una condición necesaria pero no suficiente para el cumplimiento del segundo principio de la justicia. Por ello, el alcance de su objetivo explícito - asegurar condiciones de igualdad para todas las partes- sólo es posible si a la nivelación de las condiciones sociales que constituyen el punto de partida de la competencia social y a la apertura de las trayectorias laborales se agrega un principio de distribución de la riqueza y el ingreso productos de la cooperación social.

En este sentido, las desigualdades validadas por el principio de diferencia no se presentan como vías para hacer de la desigualdad misma el rasgo distintivo de una sociedad bien ordenada, sino como un recurso al servicio de una tendencia a la igualdad que avanza conforme se van compensando todas las diferencias arbitrariamente disfrutadas o sufridas ${ }^{23}$. Por ello, en el amplio horizonte del distribucionismo de Rawls, las desigualdades controladas por el principio de diferencia están al servicio de una tendencia a la igualdad que se orienta a cumplir institucionalmente con el valor moral y político de la igualdad de las personas.

El principio de diferencia es, probablemente, el concepto rawlsiano que más amplia discusión académica ha suscitado ${ }^{24}$. Esto no es en absoluto extraño, ya que se trata de uno de los más sugerentes y fértiles principios de

${ }^{22} T J$, p. 75

${ }^{23} \mathrm{La}$ tendencia a la igualdad como exigencia de compensación nos lleva necesariamente al principio de diferencia. Dice Rawls: «Habremos de ser llevados al principio de diferencia si lo que deseamos es formular el sistema social de tal modo que nadie experimente ganancias o pérdidas como resultado de su posición arbitraria en la distribución de las capacidades naturales o de su posición inicial en la sociedad, sin por ello recibir a cambio ventajas compensatorias», $T J$, p. 102.

${ }^{24}$ Cf. J. H. Wellbank, D. Snook y D. T. Mason (comps.), John Rawls and His Critics: An Annotated Bibliography, Londres, Garland, 1984; Kukathas y Pettit (comps.), Rawls: «A Theory of Justices and its Critics, Cambridge, Polity, 1990, y J. A. Corlett (comp.), Equality and Liberty. Analizing Rawls and Nozick, Londres, Macmillan, 1991. 
justicia distributiva de la tradición liberal y, en general, del pensamiento moral y político contemporáneo. Como he dicho antes, este principio define el perfil distribucionista radical de la justicia como imparcialidad, aunque desde su formulación ha suscitado una amplia y aguda discusión acerca de sus alcances distributivos ${ }^{25}$. La necesidad de precisar las características y función del principio de diferencia al interior de la teoría de la justicia como imparcialidad llevó a Rawls a ofrecer una reformulación del segundo principio de la justicia:

Las desigualdades sociales y económicas han de ser ordenadas de tal modo que: a) funcionen para el mayor beneficio de los menos aventajados, y $b$ ) estén vinculadas a oficios y posiciones abiertas a todos bajo condiciones de justa igualdad de oportunidades ${ }^{26}$.

Como puede notarse, el principio de diferencia estaría garantizado por el enunciado a) del segundo principio de la justicia, aunque éste no queda reducido a ser mera expresión del principio de diferencia, pues incluye la prescripción de la justa igualdad de oportunidades.

Si consideramos que el principio de diferencia es el equivalente de la llamada concepción general de la justicia y, en este sentido, el criterio de orientación y justificación a largo plazo de todas las instituciones de la justicia, tendríamos que conceder que ocupa un lugar superlativo en la justicia como imparcialidad. Dice Rawls:

De hecho, la concepción general es, simplemente, el principio de diferencia aplicado a todos los bienes primarios, incluyendo la libertad y las oportunidades y, en este sentido, no limitado por otras partes de la concepción especial. Esto es evidente desde nuestra temprana discusión de los principios de la justicia. Estos principios, en orden serial, constituyen [...] la forma finalmente asumida por la concepción general cuando las condiciones sociales mejoran ${ }^{27}$.

En este contexto, el principio de diferencia es el modelo de evaluación en perspectiva histórica de las sociedades bien ordenadas. La propuesta de una concepción general de la justicia no choca con la de una concepción especial, pues ésta no es más que la concreción, bajo ciertos requerimientos y limitaciones, de las intenciones y valores enunciados en aquélla. De este modo, el principio de diferencia podría ser considerado como el concepto que define los objetivos y condiciones de cualquier formulación posible de

25 Entre los autores que niegan que el principio de diferencia sea algo más que una nueva defensa de la inequidad destacan: Russell Keat y David Miller, «Understanding Justice», Political Theory, núm. 2, febrero de 1994, y Lester C. Thurow, «Toward a Definition of Economic Justice», Public Interest, núm. 31, primavera de 1973.

26 TJ, p. 83

27 TJ, p. 83 
la justicia y, en particular, de la justicia como imparcialidad. Sin embargo, el que Rawls, para el caso de la concepción especial de la justicia, no asigne al principio de diferencia una función de distribución de las libertades y derechos ciudadanos depende, en mi opinión, de que juzga que el proceso histórico de desarrollo de las modernas democracias constitucionales ha completado ya esta distribución. Lo significativo es que, bajo la conceptualización de la concepción general, esta distribución no es otra cosa que la plasmación histórica del principio de diferencia.

En cualquier caso, su identificación con la concepción general permite atribuirle el carácter de principio universal de distribución de bienes primarios ${ }^{28}$. Si, como aquí se ha sugerido, es posible concebir la elección de principios de justicia diferentes a los de la concepción especial siempre y cuando uno no se sitúe en la perspectiva de las democracias constitucionales modernas, entonces lo que invariablemente regirá esas elecciones alternativas seguirá siendo el principio de diferencia.

Sin embargo, conceder al principio de diferencia en su dimensión histórica el papel de indicador de los acomodos empíricos de los contenidos de la justicia implicaría introducir una noción metafísica difícilmente defendible para la interpretación histórica. Tengo para mí que el galimatías de esta universalización del principio de diferencia proviene, más que de la intención de mantener la vigencia de este concepto para niveles explicativos distintos, de la conversión de la que acaso es la principal categoría igualitarista de Rawls en una metáfora de los procesos de distribución de derechos, libertades y riqueza a lo largo de la historia moderna.

En cualquier caso, no creo que existan evidencias históricas suficientes para amparar la hipótesis de que las sociedades modernas han configurado las instituciones y valores de la ciudadanía como el desenvolvimiento de un principio distributivo como el de diferencia; pero tampoco creo que el principio de diferencia pueda incluso ser coherentemente formulado como un principio distributivo general de los contenidos de la propia justicia como imparcialidad, es decir, de la concepción especial de la justicia.

En esta línea de interpretación podrían considerarse equívocas otras lecturas, también amparadas en ambigüedades de Rawls, que asignan al principio de diferencia la función distributiva de todos los bienes primarios de naturaleza económica, concediendo a éste parte de lo que antes había sido adjudicado a la justa igualdad de oportunidades. Siguiendo en algún sentido al propio Rawls, esta posición sostendría que el principio de diferencia paliaría el problema de la distribución desigual de la riqueza, el ingreso, los

\footnotetext{
28 Para una evaluación crítica del tratamiento rawlsiano de los bienes primarios y de las dificultades que genera para una concepción de la igualdad no reducida a condiciones socioeconómicas, véase Jesús Rodríguez Zepeda, «Tras Rawls: el debate de los bienes primarios, el bienestar y la igualdad», en Revista Internacional de Filosofía Política, núm. 23, junio de 2004, Barcelona, Anthropos.
} 
poderes y las prerrogativas de autoridad ${ }^{29}$. Esto parece implicar que todas las diferencias económicas significativas pueden ser reguladas por el principio de diferencia. Un ejemplo de este tipo de lectura sería la de Kymlicka, para quien la incapacidad rawlsiana para ofrecer respuesta satisfactoria al problema de los casos de discapacidad o enfermedad crónica proviene, específicamente, de la definición que hace el principio de diferencia de las posiciones peor situadas sólo en términos de posesión de bienes primarios sociales y no en términos de bienes primarios naturales ${ }^{30}$.

La objeción que puede enderezarse contra esta perspectiva sería la de que se está escamoteando al mecanismo de la justa igualdad de oportunidades una función distributiva en el terreno económico. Aunque esto sólo parecería la constatación de un problema menor, creo que sus consecuencias revisten mayor importancia que las de solapar el campo de cobertura de una categoría con el de otra. Si la justa igualdad de oportunidades se ha identificado con la llamada igualdad liberal y, seguidamente, con el diseño institucional y legal del Estado de bienestar, sostener que la función realmente distributiva sólo correspondería al principio de diferencia equivaldría a negar a la igualdad liberal el papel que tradicionalmente ha jugado como modelo reformista y nivelador de la estructura económica. Además, si Rawls ha sostenido que el principio de diferencia sólo se formula para enfrentar las diferencias que la igualdad de oportunidades inevitablemente permite que subsistan, es decir, las derivadas de las diferencias naturales entre los sujetos, tendrá que concebirse la existencia de desigualdades sociales que hayan podido ser reducidas a través de los mecanismos de la igualdad de oportunidades. En cualquier caso, ha sido el propio Rawls el que con un uso poco cuidadoso del principio de diferencia ha suscitado estas innecesarias complejidades en la discusión.

Ciertamente Rawls ha alentado las interpretaciones generalizantes del principio de diferencia al identificarlo con la concepción general de la justicia; sin embargo, también es cierto que el único tratamiento conceptual acuciosamente desarrollado por él es el relativo a su función como regla de asignación de determinados bienes primarios en el contexto de una insuficiencia distributiva de la justa igualdad de oportunidades. Por esta razón, también es posible - y valdría decir que incluso más coherente con el propio discurso de Rawls - entender el principio de diferencia «sólo» como una regla de compensación de las diferencias sociales derivadas de la desigual distribución de capacidades y talentos naturales, aunque esto, evidentemente, limitara de raíz la asignación a este principio de un estatuto histórico de dudosa consistencia. Aún más, el principio de diferencia puede ser mejor ponderado si su campo de aplicación se restringe al de un sistema de com-

\footnotetext{
${ }^{29}$ Cf. $T J$, p. 93.

${ }^{30}$ Cf. Will Kymlicka, op. cit., pp. 69-70. Para seguir este debate, véase mi artículo referido en la nota 28
} 
pensación laboral, fiscal y salarial, es decir, a la distribución de bienes primarios que no ha podido ser saldada con la aplicación de la justa igualdad de oportunidades.

Esta limitación del supuesto alcance general del principio de diferencia cumpliría el requisito de la prioridad que respecto de él mantiene la igualdad de oportunidades. Según Rawls,

es evidente que el papel del principio de justa oportunidad es uno de justicia puramente procedimental. A menos de que sea satisfecho, la justicia distributiva no puede ser dejada a cargo de sí misma, incluso dentro de un ámbito restringido ${ }^{31}$.

$\mathrm{Si}$ se considera que un modelo de justicia puramente procedimental requiere que la regularidad del funcionamiento de las reglas esté soportada por un sistema de instituciones justas que no puede ser sostenido al margen de esa regularidad, tendrá que concluirse que la vigencia de una justa igualdad de oportunidades implica un mínimo de bienes sociales primarios ampliamente distribuidos entre las posiciones representativas. Esto se debe a que la justicia puramente procedimental es presentada como una lógica de funcionamiento institucional que mantiene un equilibrio constante entre la regularidad de la aplicación de los principios «morales» que la sostienen y la certidumbre acerca de los resultados que pueden esperarse de esta aplicación. En este sentido, la justa igualdad de oportunidades no puede ser considerada sólo como una condición de igualdad formal (aunque la subsuma) para el posterior funcionamiento de un principio distributivo, sino como la aplicación regular de principios que son de suyo distributivos y que, en este sentido, garantizan de entrada la disminución de muchas de las desigualdades propias de una sociedad injusta, es decir, de una sociedad donde las desigualdades son arbitrarias.

En consecuencia, el momento lexicográfico de la justa igualdad de oportunidades implica la vigencia de dos esferas distributivas cuyos requerimientos deben ser secuencialmente satisfechos: una distribución igualitaria de los derechos y libertades garantizados por el primer principio de la justicia y una justa distribución de las oportunidades de acceso a las trayectorias laborales y las posiciones privilegiadas. Esta última esfera distributiva presupone la eliminación de todas las instituciones y diferencias sociales que limitan el libre desarrollo y capacidad de competencia profesional de las posiciones menos privilegiadas. Aún más, el requisito secuencial de que la aplicación del principio de diferencia se haga sólo hasta haber sido cumplida la condición de la justa igualdad de oportunidades mantiene la exigencia de una distribución equitativa de todos los bienes sociales de naturaleza económica que facilitan el libre acceso a las trayectorias profesionales. Esto significa que instituciones como la educación pública y gratuita, el derecho universal

${ }^{31} T J$, p. 87 
a la salud, las políticas de la llamada acción afirmativa o discriminación positiva y los programas de desarrollo comunitario, entre otras, que manifiestan con claridad la vigencia de normas distributivas de justicia, estarían bajo la cobertura de la igualdad de oportunidades y no del principio de diferencia.

Esta segunda interpretación, la de la limitación del alcance distributivo del principio de diferencia, es la que mejor coincide con los desarrollos explícitos de la idea de justicia distributiva en la teoría de la justicia como imparcialidad. Cuando Rawls define los campos de distribución al interior de una sociedad bien ordenada, queda claro cuáles habrán de ser los bienes sociales distribuidos por el principio de diferencia. Cito in extenso:

... considero que la estructura básica se halla regulada por una constitución justa que asegura las libertades de la ciudadanía equitativa [...]. Considero, también, que existe una justa (como opuesta a la formal) igualdad de oportunidades. Esto significa que, además de mantener las formas usuales de gasto social relativo al capital, el gobierno intenta asegurar la igualdad de oportunidades educativas y culturales para las personas similarmente dotadas y motivadas, ya sea a través del subsidio a las escuelas privadas o del establecimiento de un sistema educativo público. Esto permite reforzar y garantizar la igualdad de oportunidades en las actividades económicas y en la libre elección laboral. Esto se logra por medio de la supervisión de la conducta de las empresas y las asociaciones privadas y por la prohibición del establecimiento de restricciones monopólicas y barreras para el acceso a las posiciones más atractivas. Finalmente, el gobierno garantiza un mínimo social a través tanto de los gastos familiares como de los pagos especiales por enfermedad y desempleo $o$, más sistemáticamente, a través de medidas como el suplemento diferenciado al ingreso (el así llamado impuesto negativo) ${ }^{32}$.

Como puede notarse, el principio de diferencia sólo tendría vigencia en el campo específico de las posiciones laborales, los suplementos salariales y determinadas políticas fiscales, puesto que bienes sociales como la educación o el acceso libre a las ocupaciones ya estaría equitativamente distribuido por la igualdad de oportunidades. Esto podría dar la impresión de que, dado que el campo de aplicación del principio de diferencia es más bien limitado, lo prudente sería reconsiderar su supuesto perfil distribucionista radical. No obstante, creo que este perfil puede mantenerse sin demasiadas dificultades, toda vez que la función compensatoria que cumple este principio permite una redefinición cualitativa de lo que debe ser un Estado de bienestar. En efecto, la idea de que las desigualdades de riqueza e ingreso provenientes de diferencias en habilidades y talento pueden ser consideradas como injustas dado su origen arbitrario lleva a Rawls a la defensa de un sistema de compensaciones referido al merecimiento moral de los individuos y no simplemente a sus cualidades personales.

${ }^{32} T J$, p. 275. La cursiva es mía. 
Es bajo esta perspectiva que el principio de diferencia formula la exigencia moral de diseñar las instituciones sociales de tal modo que compensen las desventajas no voluntarias que la educación, el libre mercado laboral o los programas de desarrollo social no pueden por sí mismos compensar. En cualquier caso, el alcance distributivo del principio de diferencia, por limitado que sea, no hace sino redondear el alcance distributivo del segundo principio de la justicia en su conjunto. La equivalencia política e institucional de esta pareja de reglas distributivas sería la reforma del Estado de bienestar en la vía de no sólo garantizar la promoción de los sujetos desfavorecidos para permitir una libre competencia laboral, sino de dotarlos además de los recursos necesarios para que, en el marco de una estratificación social irreductible, puedan alcanzar los objetivos particulares a los que tienen derecho como personas morales libres e iguales.

Si consideramos el enfoque sistemático de la justicia como imparcialidad, y si concedemos que la discusión acerca del alcance distributivo del principio de diferencia y la igualdad de oportunidades sólo adquiere sentido cuando se plantea a propósito de la discusión sobre el grado de alejamiento de Rawls respecto de la tradición canónica del Estado de bienestar, entonces habría que conceder que la cualidad distributiva de la justicia como imparcialidad debe evaluarse en el efecto de conjunto de la aplicación combinada de los dos principios.

La teoría rawlsiana de la justicia no es el resultado de la suma de una concepción naturalista-garantista de los derechos ciudadanos y una concepción distribucionista de la riqueza y el ingreso, sino el reclamo «convencionalista» de que cualquier repartición de bienes políticos o económicos debe ser aprobado por el tribunal moral de los principios de libertad e igualdad. Se trata, en este sentido, de una teoría en la que todas las categorías significativas están al servicio de una intención distributiva, por lo que la «transferencia» de campos de aplicación entre un principio y otro no implica una desestructuración del carácter sistemático de la teoría.

Contemplada esta contextualización, es razonable sostener que el principio de diferencia sería válido, no para regular correcciones particulares en las transacciones privadas o en las relaciones laborales discretas, sino como principio inspirador de la estrategia distributiva y la política económica de una sociedad determinada. Como dice Rawls,

... los principios de la justicia, y el principio de diferencia en particular, se aplican a los principios y políticas públicos fundamentales que regulan las desigualdades sociales y económicas. Trabajan para ajustar el sistema de derechos e ingresos y para equilibrar los estándares familiares cotidianos y las reglas empleadas por este sistema. El principio de diferencia se aplica, por ejemplo, a la gravación fiscal del ingreso y la propiedad y a la política fiscal y económica ${ }^{33}$.

\footnotetext{
${ }^{33}$ John Rawls, Political Liberalism, pp. 282-283.
} 
La limitación del principio de diferencia al terreno económico y fiscal sólo es concebible si se dan por garantizadas dos distribuciones equitativas de primera importancia: la de libertades y la «justa» de oportunidades. Así, los principios rawlsianos de la justicia, en su conjunto, siguen exhibiendo una identidad distributiva radical, aunque esta radicalidad tenga que ser definida sólo en dos vías: una, como el intento de reducir al mínimo las diferencias económicas a sabiendas de que será imposible hacerlas desaparecer; la otra, como la certeza de que la eliminación de las desigualdades arbitrarias y el control sobre las que son accesibles al consenso es todo lo que se podría exigir a un proyecto moral y político razonable en el marco de una sociedad capitalista.

A fin de cuentas, el difícil equilibrio buscado por la obra de Rawls consiste en ofrecer el modelo de una sociedad bien ordenada y altamente distributiva sin tener que abogar por la desaparición del modelo de producción capitalista. Creo que en este equilibrio reside la capacidad de interpelación de la justicia como imparcialidad; y en ella residen también muchas de sus limitaciones. 